\title{
Prevalence of Clonal Complexes and Virulence Genes among Commensal and Invasive Staphylococcus aureus Isolates in Sweden
}

\author{
Gunlög Rasmussen ${ }^{1,5^{*}}$, Stefan Monecke ${ }^{2,3}$, Ralf Ehricht ${ }^{2}$, Bo Söderquist ${ }^{1,4,5}$
}

1 Department of Infectious Diseases, Örebro University Hospital, Örebro, Sweden, 2 Alere Technologies GmbH, Jena, Germany, 3 Institute for Medical Microbiology and Hygiene, TU Dresden, Dresden, Germany, 4 Laboratory Medicine, Clinical Microbiology, Örebro University Hospital Sweden, Örebro, Sweden, 5 Faculty of Medicine and Health, Örebro University, Örebro, Sweden

\begin{abstract}
Staphylococcus aureus encodes a remarkable number of virulence factors which may contribute to its pathogenicity and ability to cause invasive disease. The main objective of this study was to evaluate the association between $S$. aureus invasiveness and bacterial genotype, in terms of the presence of virulence genes and affiliation to clonal complexes. Also, the significance of different virulence genes, mainly adhesins, for the development of infective endocarditis was investigated.

DNA microarray technology was used to analyze $134 \mathrm{~S}$. aureus isolates, all methicillin-susceptible, derived from three groups of clinically well-characterized patients: nasal carriers $(n=46)$, bacteremia $(n=55)$, and bacteremia with infective endocarditis $(n=33)$.

Invasive isolates were dominant in four of the major clonal complexes: 5, 8, 15, and 25. Of the 170 virulence genes examined, those encoding accessory gene regulator group II (agr II), capsule polysaccharide serotype 5 (cap5), and adhesins such as $S$. aureus surface protein $G$ (sasG) and fibronectin-binding protein $B$ ( $f(n B B)$ were found to be associated with invasive disease. The same was shown for the leukocidin genes lukD/lukE, as well as the genes encoding serine protease $\mathrm{A}$ and $\mathrm{B}(\mathrm{sp} / \mathrm{A} / \mathrm{sp} / \mathrm{B})$, staphylococcal complement inhibitor (scn) and the staphylococcal exotoxin-like protein ( $s e t C$ or selX). In addition, there was a trend of higher prevalence of certain genes or gene clusters (sasG, agr II, cap5) among isolates causing infective endocarditis compared to other invasive isolates. In most cases, the presence of virulence genes was linked to clonal complex affiliation.

In conclusion, certain S. aureus clonal lineages harboring specific sets of virulence genes seem to be more successful in causing invasive disease.

Citation: Rasmussen G, Monecke S, Ehricht R, Söderquist B (2013) Prevalence of Clonal Complexes and Virulence Genes among Commensal and Invasive Staphylococcus aureus Isolates in Sweden. PLoS ONE 8(10): e77477. doi:10.1371/journal.pone.0077477

Editor: Karsten Becker, University Hospital Münster, Germany

Received June 24, 2013; Accepted September 2, 2013; Published October 9, 2013

Copyright: (c) 2013 Rasmussen et al. This is an open-access article distributed under the terms of the Creative Commons Attribution License, which permits unrestricted use, distribution, and reproduction in any medium, provided the original author and source are credited.

Funding: Funding for this study was provided by grants from the Research Comitte of the County Council of Örebro University Hospital. The funders had no role in study design, data collection and analysis, decision to publish, or preparation of the manuscript.

Competing interests: Stefan Monecke and Ralf Ehricht are employees at Alere Technologies GmbH, Jena, Germany and the corresponding author was asked to declare this affiliation. Although two of the co-authors are employees at Alere Technologies $\mathrm{GmbH}$, this company was not a commercial funder of this research since funding for the study was totally provided by grants from the Research Comitte of the County Council of Örebro University Hospital. Consequently: This does not alter the authors' adherence to all the PLOS ONE policies on sharing data and materials.

*E-mail: gunlog.rasmussen@orebroll.se
\end{abstract}

\section{Introduction}

S. aureus commonly colonizes the human skin and mucosal membranes without causing disease. However, it is also a wellknown pathogen causing a broad spectrum of infections ranging from superficial skin infections to invasive disease such as life-threatening bacteremia and infective endocarditis (IE). An increased incidence of $S$. aureus bacteremia has been reported [1], and it constitutes the most common etiology of IE in Sweden with increased incidence in recent years (unpublished data from the Swedish national infective endocarditis quality register). The bacterium produces several virulence factors which may contribute to its invasive potential [2], including surface-associated adhesins such as MSCRAMMs (Microbial Surface Components Recognizing Adhesive Matrix Molecules) as well as secreted virulence factors like exotoxins and enzymes. Capsular polysaccharides and regulators may also contribute to pathogenicity $[2,3]$. Since $S$. aureus is a commensal and acts as an opportunistic pathogen, the predisposing factors of the host, together with 
virulence factors of the microorganism might play a significant role for invasiveness.

Previous studies have investigated the importance of $S$. aureus clonality for invasive disease [4,5], as well as associations between different virulence genes and $S$. aureus disease [6-12], though the results have shown some contradictions. MSCRAMMs are among the factors of interest, as they are known to have the capacity to bind extra-cellular matrix proteins such as fibrinogen, fibronectin, and elastin, all of which are potentially important for the invasive capacity of $S$. aureus. More specifically, the association of adhesins such as clumping factors (Clf) and fibronectin-binding proteins (FnbP) and development of IE has previously been examined, although mainly in experimental studies [13-15]. Still, additional clinical research is needed to investigate the significance of bacterial genotype for $S$. aureus disease.

The present study aimed to evaluate the potential association between $S$. aureus invasive disease and bacterial genotype in terms of the presence of genes encoding adhesins and other virulence factors as well as affiliation to clonal complexes (CCs). Furthermore, it sought to investigate whether the prevalence of certain MSCRAMM genes is associated with $S$. aureus IE. To achieve these aims, we used DNA microarray technology to analyze $S$. aureus isolates derived from three groups of clinically well-characterized patients: nasal carriers, bacteremia, and bacteremia with IE.

\section{Material and Methods}

\section{Ethics statement}

The study was conducted in accordance with the ethical guidelines of Declaration of Helsinki and was approved by the regional ethical committee in Örebro (Dnr 543/88) and Uppsala (Dnr 2011/3349). Written informed consent to participate was provided from all patients comprising the bacteremia group without IE (Dnr 543/88). Regarding the IE patients, the regional ethical committee in Uppsala approved the use of these clinical data entered in the Swedish quality register of infective endocarditis (Dnr 2011/3349). These IE patients were written informed that clinical data were recorded in a national quality register and could be used for research purposes.

Nasal isolates were obtained from swabs of anonymized patients intended for elective orthopedic surgery who were verbally informed regarding the purpose according to a Chairman decision of the regional ethical committee in Örebro 1992. For the IE patients and the anonymized patients whose nasal swabs were used, the regional ethical committee waived the need for written consent.

\section{Bacterial isolates}

A total of 134 methicillin-susceptible $S$. aureus (MSSA) were included in the study: 46 nasal carriage and 88 bacteremic isolates. Within the bacteremia group, 33 isolates originated from patients with IE. The isolates were evaluated according to origin (nasal carriage, bacteremia with and without IE).

All isolates were derived from patients treated at Örebro University Hospital, and included in the study after ethical approval. Most of the bacteremic isolates (61/88) and corresponding clinical data were prospectively collected from hospitalized patients with a diagnosis of $S$. aureus bacteremia during 1988-1992 [16]. This group included 6 patients with concomitant IE. An additional $27 \mathrm{~S}$. aureus blood isolates from patients with IE were also included. These isolates originated from patients who had been treated for $S$. aureus IE during 2008-2011 and registered in the Swedish national IE quality register, a national database recording relevant clinical data, diagnostic procedures, treatment, and outcome. Nasal carriage isolates were obtained from nasal swabs of patients intended for elective orthopedic surgery during 1992. For each patient, only one episode of $S$. aureus bacteremia was included, with the exception of one patient who had two separate episodes of bacteremia with genotypically different isolates.

The $S$. aureus isolates were identified by routine microbiological methods such as coagulase and DNase tests. Isolates were stored at $-70^{\circ} \mathrm{C}$ in preservation medium (yeast extract; Difco Laboratories, Sparks, MD, USA; and horse serum added to trypticase soy broth; BBL, Sparks, MD, USA).

\section{Definitions}

The diagnosis of $S$. aureus bacteremia was verified by at least one positive blood culture performed with the Bactec system (Becton Dickinson, USA)In order to evaluate the importance of different patient characteristics, the patients' medical records were carefully surveyed for underlying conditions such as diabetes mellitusrenal insufficiency requiring hemodialysis or peritoneal dialysis, present malignant diseaseand immunosuppression due to treatment with corticosteroids, chemotherapylE: infective endocarditisFnbP: fibronectin-binding proteinsMLST: multilocus sequence typingCCs: clonal complexesCP: capsular polysaccharidePIA: polysaccharide intercellular adhesionSTs: sequence types or immunosuppressive disease such as leukemia. Presence of indwelling catheters (e.g. central venous catheter), pacemakers, cardiac valves, prosthetic joint devices, and osteosynthesis material were recorded. In addition we registered hematogenous complications such as IE, acute osteomyelitis, septic arthritis, deep seated abscesses, and meningitis. IE was defined according to the modified Duke criteria [17].

\section{DNA microarray based genotyping}

Genotyping was performed with the Alere StaphyType DNA microarray test (Alere Technologies $\mathrm{GmbH}$, Jena, Germany), according to protocols and procedures described in detail elsewhere $[18,19]$. This DNA microarray includes 333 target sequences corresponding to approximately 170 distinct genes and their allelic variants, covering species markers, markers for the recognition of $\mathrm{SCCmec}$, and capsule types as well as agr groups, resistance genes, exotoxins, MSCRAMM genes, and others. Primer and probe sequences have been published previously $[19,20]$.

In short, monoclonal $S$. aureus cultures were grown on Columbia blood agar overnight at $37^{\circ} \mathrm{C}$. Harvested cultures were enzymatically lysed using lysozyme, lysostaphin, and RNAse. DNA was prepared using commercially available spin columns (Qiagen, Hilden, Germany). The resulting RNA-free 
Table 1. Characteristics of patients with S. aureus bacteremia with or without IE.

\begin{tabular}{|c|c|c|c|}
\hline Clinical characteristics & Bacteremia IE $n=33(\%)$ & Bacteremia non IE $n=55(\%)$ & Bacteremia all $n=88(\%)$ \\
\hline Median age & 56 (range 24-91) & 66 (range 10-91) & 63 (range 10-91) \\
\hline Sex (male) & $18(55)$ & $34(62)$ & $52(59)$ \\
\hline Patients with $\geq 2$ blood cultures positive for $S$. aureus & $31(94)$ & $47(85)$ & $78(89)$ \\
\hline Immunosuppression $^{1}$ & $3(9)$ & $5(9)$ & $8(9)$ \\
\hline Diabetes mellitus $^{2}$ & $2(6)$ & $9(16)$ & $11(13)$ \\
\hline Malignant disease & $2(6)$ & $7(13)$ & $9(10)$ \\
\hline IV drug abuse & $12(36)$ & $1(2)$ & $13(15)$ \\
\hline Indwelling devices ${ }^{3}$ & $8(24)$ & $12(22)$ & $20(23)$ \\
\hline Hematogenous complications ${ }^{4}$ & $33(100)$ & $25(45)$ & $58(66)$ \\
\hline Acute osteomyelitis & 1 (3) & $14(25)$ & $15(17)$ \\
\hline Acute septic arthritis & $6(18)$ & $9(16)$ & $15(17)$ \\
\hline Meningitis & $1(3)$ & $2(4)$ & $3(3)$ \\
\hline Other CNS embolization & $5(15)$ & $0(0)$ & $5(6)$ \\
\hline Mortality $^{5}$ & $3(9)$ & $3(5)$ & $6(7)$ \\
\hline
\end{tabular}

1. Treatment with corticosteroids, chemotherapy or immunosuppression due to leukemia

2. Treated with insulin or oral antidiabetics

3. Prescence of central venous catheter $(n=2)$, pacemaker, cardiac valves, prosthetic joint devices or osteosynthesis material

4. IE, acute osteomyelitis, septic arthritis, deep seated abscesses or meningitis

5. Within 12 weeks after the initial positive blood culture

doi: 10.1371/journal.pone.0077477.t001

unfragmented DNA preparations were used as templates in a multiplexed primer elongation with only a single reverse primer per target. During that step, biotin-16-dUTP was incorporated into the amplicons. Labeled amplicons were hybridized to the microarray. After washing and blocking steps, horseradishperoxidase-streptavidin conjugate was added. Following further incubation and washing, hybridizations were visualized using a precipitating dye. An image of the microarray was recorded using a designated reader (Alere Technologies $\mathrm{GmbH}$, Jena, Germany). Normalized intensities of the spots were calculated based on their average intensities and further analyzed as described previously [20].

The assignment of isolates to CCs or sequence types (STs), as defined by multilocus sequence typing (MLST) [21], was determined by an automated comparison of hybridization profiles to a collection of reference strains previously subjected to MLST [19].

\section{Statistics}

Proportions were compared with Fisher's exact test, using version 17 of the SPSS software package. $P$-values $<0.05$ were considered statistically significant.

\section{Results}

\section{Patient characteristics}

Clinical data for patients with bacteremia, with and without IE, are shown in Table 1. No clinical data were available for the nasal carriage population. All 33 patients with IE fulfilled the criteria of definite IE according to the modified Duke criteria [17]. Of patients with IE, 19 of $33(58 \%)$ had one or more factors predisposing for IE (underlying heart disease $n=5$; prosthetic heart valves $n=3$; previous history of IE $n=4$; injection drug use $n=12$ ). All patients except one were examined by echocardiography (28 transesophageal and 4 transthoracic). The diagnosis was confirmed by autopsy in two patients, one of whom did not undergo echocardiographic investigation. Twenty-three patients showed vascular embolization (osteomyelitis $n=1$; septic arthritis $n=6$; embolization to central nervous system/meningitis $n=6$; septic pulmonary infarcts $n=11$; skin and soft tissue $n=4$ ). Eight patients underwent surgical intervention due to IE, and four patients relapsed shortly after the end of treatment or operation.

\section{Assignment to CCs and STs according to DNA microarray analysis}

The assignment of $134 \mathrm{~S}$. aureus isolates to CCs and STs is shown in Table 2. The isolates displayed $18 \mathrm{CCs} / \mathrm{STs}$. Six CCs dominated and accounted for 113 of the 134 isolates (84\%), each comprising 9 to 36 isolates. Four of the major CCs $(5,8,15,25)$ included 46 isolates, 37 invasive and 9 carriage isolates, and this difference was statistically significant $(p=0.012)$. Moreover, within CCS 5,7 of 11 isolates $(64 \%)$ originated from patients with IE. Counting on significance level, isolates within the four major CCs were combined, considering the relatively limited number in each $\mathrm{CC}$. In contrast, $S$. aureus carriage within CC30 accounted for 18 of the 31 isolates $(p=0.002)$. The 36 isolates in CC45 showed no difference between the isolates obtained from the carriage and invasive groups. The remaining $12 \mathrm{CCs} / \mathrm{STs}$ comprised 21 of 134 isolates (16\%), each CC/ST represented by 1-5 isolates each. ST34 was analyzed separately from CC30 to which it belongs according to MLST based clustering because of distinct 
Table 2. Distribution of CCs and ST.

\begin{tabular}{|c|c|c|c|c|c|}
\hline Clonal complex & Isolates No within CC & Nasal carriage (\%) & Invasive (\%) & Odds ratio $(95 \% \mathrm{Cl})$ & $p$-value ${ }^{1}$ \\
\hline 5 & $11^{2}$ & $1(9)$ & $10(91)$ & $3.0(1.3-6.9)^{3}$ & $0.012^{3}$ \\
\hline 8 & 9 & $2(22)$ & $7(78)$ & & \\
\hline 15 & 15 & $3(20)$ & $12(80)$ & & \\
\hline 25 & 11 & $3(27)$ & $8(73)$ & & \\
\hline 30 & 31 & $18(58)$ & $13(42)$ & $0.25(0.11-0.57)$ & 0.002 \\
\hline 45 & 36 & $14(39)$ & $22(61)$ & $0.72(0.32-1.6)$ & 0.42 \\
\hline other $^{4}$ & 21 & $5(24)$ & $16(76)$ & na & na \\
\hline Total & 134 & $46(34)$ & $88(66)$ & & \\
\hline
\end{tabular}

1. Fisher's exact test

2. $7 / 11$ isolates originated from patients with IE

3. Counted on $\operatorname{CCS} 5,8,15$ and 25 together

4. ST34, CC1, CC9, CC12, CC20, CC22, ST49, CC50, CC59, CC97, CC182, CC395 na; not applicable

Bold figures; statistically significant

doi: 10.1371/journal.pone.0077477.t002

Table 3. Distribution of agr groups.

\begin{tabular}{|c|c|c|c|c|c|}
\hline agr & Isolates n=134 (\%) & Nasal carriage $n=46(\%)$ & Invasive $\mathrm{n}=\mathbf{8 8}(\%)$ & Odds ratio $(95 \% \mathrm{Cl})$ & p-value ${ }^{1}$ \\
\hline$\overline{12}$ & $62(46)$ & $21(46)$ & $41(47)$ & $1.0(0.51-2.1)$ & 1 \\
\hline$\|^{3}$ & $31(23)$ & $5(11)$ & $26(30)$ & $3.4(1.2-9.7)$ & 0.017 \\
\hline $1 \mathrm{II}^{4}$ & $35(26)$ & $18(39)$ & $17(19)$ & $0.37(0.17-0.82)$ & 0.022 \\
\hline $\mathrm{IV}^{5}$ & $6(4)$ & $2(4)$ & $4(5)$ & $1.0(0.19-5.9)$ & 1 \\
\hline \multicolumn{6}{|c|}{ 1. Fisher's exact test } \\
\hline \multicolumn{6}{|c|}{ 2. CC8, CC20, CC22, CC25, CC45 (except CC45 agr IV), CC59, CC97, CC182, ST 426} \\
\hline \multicolumn{6}{|c|}{ 3. CC5, CC9, CC12, CC15, ST49 } \\
\hline \multicolumn{6}{|c|}{ 4. CC1, СC30, ST34 } \\
\hline \multicolumn{6}{|c|}{ 5. CC45 agr IV, CC50 } \\
\hline \multicolumn{6}{|c|}{ Bold figures; statistically significant } \\
\hline doi: & 371/journal.pone.0077 & & & & \\
\hline
\end{tabular}

characteristics (absence of cna, presence of seh). Of the 2 isolates within ST34, both originated from IE patients.

\section{Agr groups}

The distribution of isolates among agr groups I-IV according to carrier status and invasive disease is shown in Table 3, along with affiliation to CCs. Affiliation to agr groups was strongly linked to bacterial clonality in that all isolates assigned to a specific CC clustered to the same agr group (Table 3). A total of $46 \%(62 / 134)$ of the MSSA isolates were assigned to agr group I and only 6 isolates were assigned to agr group IV. Neither group showed differences regarding the clinical origin of bacterial isolates. However, within agr group II, 26 of 31 isolates were of invasive origin and 13 of these were from patients with IE, but in agr group III, 18 of 35 isolates were carriage isolates. Thus, agr group II was associated with invasive disease $(p=0.017)$, while agr group III was linked with carriage status $(p=0.022)$.

\section{Surface-associated polysaccharides}

All isolates carried capsular polysaccharide (CP) serotype 5 $(29 \%)$ or $8(71 \%)$; no CP1 was found. There was an association between invasiveness and CP5 ( $p=0.044) ; 15$ of the 39 CP5 isolates (38\%) were from patients with IE. The presence of cap genes was in accordance with affiliations to CCs (Figure 1), but there were no correlation between cap genes and agr groups.

All isolates harbored the genes encoding polysaccharide intercellular adhesion (PIA); icaA, icaC, and icaD.

\section{MSCRAMMs}

Genes encoding 15 different MSCRAMMs were analyzed (Table 4), and two of these ( $f n b B$ and sasG) were more prevalent among invasive isolates than carriage isolates ( $p=0.008$ and $p=0.004$, respectively), while the converse was observed for cna $(p=<0.0001)$. Carriage of sas $G$ and cna was in concordance with $C C$ affiliation (Figure 1). The genes $b b p$, ebh, map, and sdrD were found in most isolates (88-99\%), regardless of clinical origin, while the remaining genes (clfA, clfB, ebpS, eno, fib, fnbA, sdrC, and $v w b$ ) were present in all isolates. In addition, bap, encoding a biofilm-associated protein was abscent in all isolates. 
Table 4. Presence of virulence genes.

\begin{tabular}{|c|c|c|c|c|c|}
\hline Virulence gene & Product & Nasal carriage $n=46(\%)$ & Invasive $n=88(\%)$ & Odds ratio $(95 \% \mathrm{Cl})$ & p-value ${ }^{1}$ \\
\hline \multicolumn{6}{|l|}{ Exopolysaccharides } \\
\hline cap5 & capsular polysaccharide 5 & $8(17)$ & $31(35)$ & $2.6(1.1-6.2)$ & 0.044 \\
\hline cap8 & capsular polysaccharide 8 & $38(83)$ & $57(66)$ & $0.39(0.16-0.93)$ & 0.044 \\
\hline$i c a A, D, C$ & polysaccharide intercellular adhesin & $46(100)$ & $88(100)$ & & 1 \\
\hline \multicolumn{6}{|l|}{ MSCRAMM genes } \\
\hline$b b p$ & Bone sialoprotein-binding protein & $39(85)$ & $79(90)$ & $1.6(0.55-4.55)$ & 0.41 \\
\hline clfA, clfB & Clumping factors $\mathrm{A}$ and $\mathrm{B}$ & $46(100)$ & $88(100)$ & & \\
\hline cna & Collagen binding adhesin & $37(80)$ & $43(49)$ & $0.23(0.10-0.54)$ & $<0.0001$ \\
\hline$e b h$ & Cell wall associated fibronectin-binding protein & $44(96)$ & $86(98)$ & $2.0(0.27-14)$ & 0.61 \\
\hline ebps & Cell surface elastin-binding protein & $46(100)$ & $88(100)$ & & 1 \\
\hline eno & Enolase & $46(100)$ & $88(100)$ & & 1 \\
\hline fib & Fibrinogen binding protein & $46(100)$ & $88(100)$ & & 1 \\
\hline fnbA & Fibronectin-binding protein $\mathrm{A}$ & $46(100)$ & $88(100)$ & & 1 \\
\hline$f n b B$ & Fibronectin-binding protein $\mathrm{B}$ & $27(59)$ & $71(81)$ & $2.9(1.3-6.5)$ & 0.008 \\
\hline map & Major hisocompatibility complex class II analog protein & $46(100)$ & $87(99)$ & $1.5(1.4-1.7)$ & 1 \\
\hline sasG & S. aureus surface protein $\mathrm{G}$ & $8(17)$ & $38(43)$ & $3.6(1.5-8.6)$ & 0.004 \\
\hline$s d r C$ & Serine-aspartate repeat protein C & $46(100)$ & $88(100)$ & & 1 \\
\hline$s d r D$ & Serine-aspartate repeat protein $D$ & $40(87)$ & $81(92)$ & $1.7(0.55-5.5)$ & 0.37 \\
\hline$v w b$ & Van Willebrand factor binding protein & $46(100)$ & $88(100)$ & & 1 \\
\hline \multicolumn{6}{|l|}{ Exotoxins } \\
\hline \multicolumn{6}{|l|}{ Hemolysins } \\
\hline hla & a-toxin & $46(100)$ & $86(98)$ & & 0.55 \\
\hline$h l b$ & $\beta$-toxin & $39(85)$ & $71(88)$ & $0.80(0.30-2.1)$ & 0.81 \\
\hline hld & $\delta$-toxin & $46(100)$ & $88(100)$ & & 1 \\
\hline $\mathrm{h} \mathrm{l}^{2}$ & Putative protein similar to hemolysin & $46(100)$ & $87(99)$ & & 1 \\
\hline$h I I I I^{3}$ & Channel protein, hemolysin III family protein & $45(98)$ & $87(99)$ & & 1 \\
\hline \multicolumn{6}{|l|}{ Leukocidins } \\
\hline lukF, lukS, hlgA & $\mathrm{y}$-toxin & $46(100)$ & $88(100)$ & & 1 \\
\hline lukF-PV, lukS-PV & Panton-Valentine leukotoxin & $0(0)$ & $1(1)$ & & 1 \\
\hline lukD, lukE & Leukocidin D, E component & $12(26)$ & $46(52)$ & $3.1(1.4-6.8)$ & 0.006 \\
\hline leukocidin homologue gene 4 & Leukocidin homologue family protein & $46(100)$ & $88(100)$ & & 1 \\
\hline \multicolumn{6}{|l|}{ Exfoliative toxins } \\
\hline etA & Exfoliative toxin $\mathrm{A}$ & $3(7)$ & $4(5)$ & & 0.69 \\
\hline etB & Exfoliative toxin B & $0(0)$ & $0(0)$ & & 1 \\
\hline etD & Exfoliative toxin D & $3(7)$ & $8(9)$ & & 0.75 \\
\hline \multicolumn{6}{|l|}{ Enterotoxins } \\
\hline sea & Staphylococcal enterotoxin A & $10(22)$ & $16(18)$ & $0.80(0.33-1.9)$ & 0.65 \\
\hline sea $(N 315)^{5}$ & Staphylococcal enterotoxin A, allele from N315 & $2(4)$ & $12(14)$ & & 0.14 \\
\hline seb & Staphylococcal enterotoxin B & $2(4)$ & $7(8)$ & & 0.72 \\
\hline sectsel & Staphylococcal enterotoxin $C+L$ & $7(15)$ & $12(14)$ & $0.88(0.32-2.4)$ & 0.80 \\
\hline sed+sej+ser & Staphylococcal enterotoxin $D+J+R$ & $3(7)$ & $9(10)$ & & 0.55 \\
\hline see & Staphylococcal enterotoxin E & $0(0)$ & $0(0)$ & & 1 \\
\hline egc-cluster 6 & Staphylococcal enterotoxin $\mathrm{G}+\mathrm{I}+\mathrm{M}+\mathrm{N}+\mathrm{O}+\mathrm{U}$ & $40(87)$ & $61(69)$ & $0.34(0.128-0.89)$ & 0.034 \\
\hline seh & Staphylococcal enterotoxin $\mathrm{H}$ & $1(2)$ & $4(5)$ & & 0.66 \\
\hline sek+seq & Staphylococcal enterotoxin $\mathrm{K}+\mathrm{Q}$ & $0(0)$ & $4(5)$ & & 0.30 \\
\hline tst1 & Toxic shock syndrome toxin (TSST)-1 & $15(33)$ & $15(17)$ & $0.43(0.19-0.97)$ & 0.050 \\
\hline \multicolumn{6}{|l|}{ Enzymes } \\
\hline aur & Aureolysin & $46(100)$ & $88(100)$ & & 1 \\
\hline$s p / A^{7}, s p / B^{8}$ & Serine protease A, B & $12(26)$ & $46(55)$ & $3.4(1.6-7.4)$ & 0.002 \\
\hline splE & Serine protease $\mathrm{E}$ & $29(63)$ & $50(57)$ & $0.77(0.37-1.6)$ & 0.58 \\
\hline $\operatorname{ssp} A^{9}$ & Glutamyl endopeptidase & $46(100)$ & $88(100)$ & & 1 \\
\hline$s s p B^{10}+s s p P^{11}$ & Staphopain B, A & $46(100)$ & $88(100)$ & & 1 \\
\hline sak & Staphylokinase & $38(83)$ & $73(83)$ & $1.0(0.40-2.6)$ & 1 \\
\hline chp & Chemotaxis inhibitory protein & $38(83)$ & $69(78)$ & $0.77(0.31-1.9)$ & 0.65 \\
\hline
\end{tabular}


Table 4 (continued).

\begin{tabular}{|c|c|c|c|c|c|}
\hline Virulence gene & Product & Nasal carriage $n=46(\%)$ & Invasive $n=88(\%)$ & Odds ratio $(95 \% \mathrm{Cl})$ & $p_{\text {-value }}{ }^{1}$ \\
\hline $\operatorname{sen}$ & Staphylococcal complement inhibitor & $41(89)$ & $86(98)$ & $5.2(0.98-28)$ & 0.047 \\
\hline \multicolumn{6}{|c|}{ Miscellaneous genes } \\
\hline $\operatorname{edin} A+C$ & Epidermal cell differentiation inhibitor $A+C$ & $0(0)$ & $0(0)$ & & 1 \\
\hline edinB & Epidermal cell differentiation inhibitor B & $3(7)$ & $8(9)$ & & 0.75 \\
\hline $\operatorname{set} C^{12}$ & Staphylococcal exotoxin-like protein & $25(56)$ & $70(81)$ & $3.3(1.5-7.3)$ & 0.004 \\
\hline isd $A$ & Transferrin binding protein & $46(100)$ & $88(100)$ & & 1 \\
\hline
\end{tabular}

1. Fisher's exact test

2. Locus tag SACOL0921, GenBank CP000046.1: Position 927776-928816

3. Locus tag SACOL2160, GenBank CP000046.1: Position 2239231-2239914

4. Locus tag SACOL2004, GenBank CP000046.1: Position 2064956-2065972, Locus tag SACOL2006, GenBank CP000046.1: Position 2065994-2067049

5. Also known as enterotoxin sep

6. 5 isolates belonging to $\mathrm{CC} 50$ showed a partial deletion of the locus egc-cluster missing seg

7. Locus tag SACOL1869, GenBank CP000046.1: Position 1920785-1921501

8. Locus tag SACOL1868, GenBank CP000046.1: Position 1919938-1920660

9. Locus tag SACOL1057, GenBank CP000046.1: Position 1063016-1064026

10. Locus tag SACOL1056, GenBank CP000046.1: Position 1061753-1062934

11. Locus tag SACOL1970, GenBank CP000046.1: Position 2034319-2035485

12. Locus tag SACOL0442, GenBank CP000046.1: Position 445958-446569. Two isolates with ambiguous results not included.

Bold figures; statistically significant

The distribution of genes encoding exopolysacharides, MSCRAMMs, exotoxins, enzymes and other miscellaneous genes are shown in Table 4

doi: 10.1371/journal.pone.0077477.t004

\begin{tabular}{|l|c|c|c|c|c|c|}
\hline & CC5 & CC8 & CC15 & CC25 & CC30 & CC45 \\
\hline cap 5 & 0 & 0 & $\bigcirc$ & 0 & 0 & 0 \\
\hline cap 8 & $\bigcirc$ & 0 & 0 & 0 & 0 & 0 \\
\hline cna & $\bigcirc$ & $\bigcirc$ & $\bigcirc$ & $\bigcirc$ & 0 & 0 \\
\hline fnbB & 0 & 0 & 0 & 0 & 0 & 0 \\
\hline sasG & 0 & 0 & 0 & $\bigcirc$ & 0 & 0 \\
\hline lukD/E & 0 & 0 & 0 & 0 & $\bigcirc$ & 0 \\
\hline egc-cluster & 0 & $\bigcirc$ & $\bigcirc$ & 0 & 0 & 0 \\
\hline splA/splB & 0 & 0 & 0 & 0 & 0 & 0 \\
\hline setC & 0 & 0 & 0 & 0 & $\bigcirc$ & 0 \\
\hline
\end{tabular}

Figure 1. Distribution of genes associated with invasive disease or nasal carriage status in relation to CCs. Black circle indicates that all isolates within the $\mathrm{CC}$ harbor the gene and white circle that none of the isolates does. A divided circle indicates variable gene presence within the CC. In most cases the presence of virulence genes was linked to CC affiliation.

doi: 10.1371/journal.pone.0077477.g001

\section{Genes encoding toxins}

The hemolysin genes hla, hld, hl, and hllll (loci and gene positions in Table 4) were present in all isolates except one hla-negative ST49 isolate and two hIlll-negative isolates within CC22. The hlb gene was found in $110 / 134$ isolates (82\%), with no difference between the invasive and the nasal carriage group. Of leukocidins, only one isolate carried the PVLencoding genes (lukF-PV, lukS-PV). However, lukD/lukE were more prevalent among invasive isolates (46/88) compared to nasal carriage isolates (12/46), and were thus significantly associated with invasive disease $(p=0.006)$. Genes encoding gamma-toxin (lukF/lukS/hlgA) as well as leukocidin homologue family protein (loci and gene position in Table 4) were ubiquitous.

Genes encoding exfoliative toxins were absent (etB) or rare (etA $5 \%$; etD $8 \%$ ), and all etD-positive isolates were assigned to $\mathrm{CC} 25$. The tst1-gene was found in $22 \%$ of isolates, and was more prevalent among nasal carriage isolates compared to invasive isolates $(p=0.005)$. 
Table 5. Comparison between invasive non IE isolates and IE isolates in virulence genes associated with invasive disease.

\begin{tabular}{llll}
\hline & & & \\
\hline Virulence gene & Nasal carriage $\mathbf{n = 4 6 ~ ( \% ) ~}$ & Invasive non IE $\mathbf{n = 5 5}$ (\%) & Invasive IE $\mathbf{n = 3 3}$ (\%) \\
\hline agr II & $5(11)$ & $13(24)$ & $13(39)$ \\
cap5 & $8(17)$ & $16(29)$ & $15(45)$ \\
fnbB & $27(59)$ & $47(85)$ & $24(73)$ \\
sasG & $8(17)$ & $18(33)$ & $20(61)$ \\
lukD/lukE & $12(26)$ & $28(51)$ & $20(61)$ \\
splA, splB & $12(26)$ & $28(51)$ & $20(61)$ \\
setC & $25(56)$ & $44(80)$ & $26(79)$ \\
\hline
\end{tabular}

doi: 10.1371 /journal.pone.0077477.t005

Of enterotoxin genes, those within the egc cluster (seg+sei + sem+sen+seo+seu) were the most frequent (64\%) ones, being linked to bacterial clonality (Figure 1) and significantly associated with carriage isolates $(p=0.034)$. The remaining enterotoxin genes were found in $0-19 \%$ of the isolates with no difference in prevalence with regard to clinical origin.

The presence of genes encoding proteases, $s p / A$ and $s p / B$ (loci and gene position in Table 4) was associated with invasive isolates $(p=0.002)$, but there was no difference concerning $s p l E$. SspA, sspB, and $s s p P$ (loci and gene position in Table 4) were ubiquitous among isolates. SetC (also known as selx, locus and gene position in Table 4) was also found to be associated with invasive disease $(p=0.004)$. Genes encoding HLB-converting phages (sak, chp, scn) were present in 80$95 \%$ of isolates but genes encoding epidermal cell differentiation inhibitors were absent (edinA, edinC) or rare (edinB; $8 \%$ ). All isolates harbored the gene is $d A$, encoding transferrin-binding protein.

\section{Genes associated with invasive disease}

A further comparison between invasive non-IE isolates and IE isolates was performed in virulence genes which demonstrated a significant association with invasive disease (Table 5).

\section{Discussion}

S. aureus encodes a remarkable number of surfaceassociated as well as extracellular virulence factors. However, previous studies have shown contradictory results regarding the importance of these different virulence genes for invasiveness. Based on 134 MSSA isolates from three wellcharacterized patient populations, the aim of the present study was to evaluate the potential association between $S$. aureus disease and bacterial genotype with a focus on clonality and genes encoding MSCRAMMs. We used DNA microarray based analysis, which offers the great advantage of a simultaneous and fast analysis of a large number of virulence genes as well as assignment to clonal lineages at a very reasonable cost compared to multiple PCRs or whole genome sequencing.

The $S$. aureus isolates were distributed between six major CCs, mainly in concordance with previous studies from Europe $[5,10,22]$ and the USA [4]. CC30 and CC45 comprised the largest number of isolates. Within the other four major CCs (5,
8, 15, and 25), invasive isolates dominated. In contrast, some previous studies have failed to show an association between invasive disease and clonality [5,9]. By studying less heterogeneous $S$. aureus infections, Fowler et al [4] could show an association between invasive disease with hematogenous complications and isolates within CC5 and CC30. The results of Fowler et al are in accordance with our results for CC5 but not for CC30. However, in their study it was the MRSA isolates within CC30 that were significantly associated with invasiveness. Our study only involved MSSA isolates, and CC30-MRSA are rare to virtually non-existent in Sweden. Thus epidemiological differences between the places where the studies were performed may explain this apparent discrepancy. Of isolates assigned to CC5, the majority originated from patients with IE. Although the total number of isolates within CC5 was limited, this result may suggest an association between CC5 and serious invasive $S$. aureus disease.

Due to polymorphisms in the gene encoding an autoinducing peptide in the agr locus, the $S$. aureus isolates are assigned to agr groups I to IV. As described in previous studies, the agr groups are strongly linked to bacterial clonality $[8,19]$, although both the present study and previous work [19] showed that isolates within the same agr group may belong to genetically diverse CCs. In our study, isolates within agr II were significantly associated with invasive disease, and $50 \%$ of invasive isolates within agr II originated from patients with IE. Since agr II included isolates assigned to CC5 and CC15, and invasive isolates dominated within these clones, it is not possible to conclude whether this association is a consequence of clonality or assignment to agr group. A previous study by Jarraud et al also showed a positive relationship between agr II and IE [8].

As expected, all isolates carried CP serotype 5 or 8 [23]. Among the isolates that displayed CP5, invasive isolates dominated which is consistent with a previous study in which a CP5-positive strain in a mouse model showed a higher bacteremia level as well as enhanced capacity to persist in the bloodstream, compared to a CP8-positive strain [24]. It is also worth noting the even stronger correlation between CP5 and IE. However, as for agr group, CP type is linked to the affiliation to CCs, and so the association between CP5 and invasive disease as well as IE could be a consequence of clonality. The absence of correlation between capsule serotypes and agr groups is also in line with results in a previous study [19]. 
Due to the capacity of MSCRAMMs to bind extracellular matrix proteins, the significance of MSCRAMM genes for invasive disease and IE has been thoroughly examined previously $[7,11-15,25]$. The present study showed a significant association between the presence of $\mathrm{fnbB}$ as well as sasG and invasive disease, in accordance with previous studies [26,27]. There was also a trend towards a higher prevalence of sas $G$ among isolates from IE patients compared to other invasive isolates. The presence of sas $G$, but not $f n b B$, was in accordance with affiliation to CCs.

The results are however difficult to interpret, due to the overlapping properties of many adhesins. Besides binding fibronectin, both FnbA and FnbB have the capacity to bind elastin; although unlike $\mathrm{FnbA}$, FnbB has not been shown to promote platelet aggregation [28] or bind fibrinogen. The overall capacity to bind fibronectin is not crucial based on whether the isolate carries one or both fnb genes [27]. This may indicate that the additional role of $\mathrm{FnbB}$, such as binding elastin, may be important for $S$. aureus invasive capacity. The lack of evidence for FnbB promoting platelet aggregation is consistent with our findings, which showed no difference in $f n b B$ gene prevalence between the invasive non-IE isolate group and the IE isolate group.

The SasG protein shows opposing characteristics; it prevents adhesion to extracellular matrix components such as fibronectin, cytokeratin 10 , and $\operatorname{lgG}$, while at the same time it promotes adhesion to nasal epithelial cells as well as biofilm formation [29]. The gene sas $G$ shares sequence similarity with a plasmin sensitive protein encoded by the gene $\mathrm{pls}$, in which mutation has been correlated with reduced invasion of host cells [30]. It is thus plausible that sasG also plays an important role for $S$. aureus invasiveness. Unexpectedly, and contrary to previous studies $[7,31,32]$, cna was more common in nasal carriage isolates than in invasive isolates. An explanation for this finding could be that cna is also a CC-associated marker. Since CC30 is overrepresented among carrier isolates and since all CC30 isolates also carry cna, the apparent association of this gene with carriage might be a function of the clonality of the strains in question. Furthermore, cna is the most important collagen-binding adhesin protein of $S$. aureus [33], and this feature may also be important for adherence to the nasal epithelial cells.

Several other MSCRAMM genes shown to be important for invasiveness and development of IE are highly conserved in the $S$. aureus genome [34]. This is in accordance with our results, where all isolates harbored the genes clfA, clfB, fnbA, and sdrC. On the other hand, bap, which encodes a biofilmassociated protein, was absent in all isolates, which is consistent with the fact that this gene has so far only been reported in animal strains [35].

As expected, hla was present in almost all isolates, with some occasional exceptions probably due to single mutations. Similarly, hlb was also present at high frequency, though its function depends on whether the $h l b$-converting prophage is integrated or not. In contrast to hemolysin genes, those encoding exfoliative toxins were rare, which was expected since patients with exfoliative staphylococcal disease were not specifically included in the present study. Egc was the most prevalent among enterotoxin genes, and as shown before linked to certain CCs and negatively associated with invasive disease $[10,36]$. Among leukocidins, the prevalence of lukD +lukE was high, linked to CCs and significantly associated with invasive disease, also shown by Eiff et al [37]. The $s p / A / s p / B$ genes were significantly associated with invasive disease, as well as setC.

A limitation of our study is that we have merely investigated the presence of genes harbored by $S$. aureus, rather than their expression, and that analysis was restricted to those alleles of genes covered by the DNA microarray. Moreover, specific patient-related risk factors such as immunosuppression, intravenous drug use, and indwelling medical devices may predispose for invasive $S$. aureus disease. However, we included isolates from patients with these risk factors, as it might be a reason why not all patients with predisposing medical conditions get invasive disease. Conversely, a major advantage of the study is the well-characterized patient population.

In conclusion our study indicates that invasive $S$. aureus isolates are related to certain CCs. We also found a significant association between invasiveness and genes encoding $\mathrm{CP}$ type as well as specific MSCRAMMs such as $f n b B$ and sasG. Moreover, our results suggest a trend toward even higher prevalence of certain virulence genes among isolates causing IE compared to other invasive isolates. However, in most cases the presence of virulence genes was linked to $\mathrm{CC}$ affiliation. It is also known that certain clonal lineages carry specific sets of virulence genes [20]. Even though $S$. aureus isolates from all CCs can cause invasive disease, it is reasonable to assume that certain $S$. aureus clonal lineages harboring specific sets of virulence genes are more successful at causing an invasive disease.

Against the background of increased life expectancy, implementation of more advanced medical interventions, and the emergence of antibiotic resistance, continuous surveillance of the incidence of $S$. aureus bacteremia is important. There may be continuous increase in the incidence of $S$. aureus bacteremia as well as changes in the structure and composition of the bacterial population. A study to further investigate any changes in the molecular epidemiology of invasive $S$. aureus isolates over the last 3 decades is currently ongoing.

\section{Acknowledgements}

We thank Elke Müller, Annett Reissig, and Jana Sachtschal for technical assistance with the microarray.

\section{Author Contributions}

Conceived and designed the experiments: GR BS SM RE. Performed the experiments: SM RE. Analyzed the data: GR BS SM RE. Contributed reagents/materials/analysis tools: GR BS SM RE. Wrote the manuscript: GR BS SM RE. 


\section{References}

1. Laupland KB, Lyytikäinen $O$, Søgaard M, Kennedy KJ, Knudsen JD et al. (2013) The changing epidemiology of Staphylococcus aureus bloodstream infection: a multinational population-based surveillance study. Clin Microbiol Infect 19: 465-471. doi:10.1111/j. 1469-0691.2012.03903.x. PubMed: 22616816.

2. Lowy FD (1998) Staphylococcus aureus infections. N Engl J Med 339: 520-532. doi:10.1056/NEJM199808203390806. PubMed: 9709046.

3. Ferry T, Perpoint T, Vandenesch F, Etienne J (2005) Virulence determinants in Staphylococcus aureus and their involvement in clinical syndromes. Curr Infect Dis Rep 7: 420-428. doi:10.1007/ s11908-005-0043-8. PubMed: 16225779

4. Fowler VG Jr., Nelson CL, Mclntyre LM, Kreiswirth BN, Monk A et al. (2007) Potential associations between hematogenous complications and bacterial genotype in Staphylococcus aureus infection. J Infect Dis 196: 738-747. doi:10.1086/520088. PubMed: 17674317.

5. Feil EJ, Cooper JE, Grundmann H, Robinson DA, Enright MC et al. (2003) How clonal is Staphylococcus aureus? J Bacteriol 185: 3307-3316. doi:10.1128/JB.185.11.3307-3316.2003. PubMed: 12754228.

6. Gill SR, Mclntyre LM, Nelson CL, Remortel B, Rude T et al. (2011) Potential associations between severity of infection and the presence of virulence-associated genes in clinical strains of Staphylococcus aureus. PLOS ONE 6: e18673. doi:10.1371/journal.pone.0018673. PubMed: 21541311

7. Peacock SJ, Moore CE, Justice A, Kantzanou M, Story L et al. (2002) Virulent combinations of adhesin and toxin genes in natural populations of Staphylococcus aureus. Infect Immun 70: 4987-4996. doi:10.1128/ IAI.70.9.4987-4996.2002. PubMed: 12183545.

8. Jarraud S, Mougel C, Thioulouse J, Lina G, Meugnier H et al. (2002) Relationships between Staphylococcus aureus genetic background, virulence factors, agr groups (alleles), and human disease. Infect Immun 70: 631-641. doi:10.1128/IAI.70.2.631-641.2002. PubMed: 11796592.

9. Lindsay JA, Moore CE, Day NP, Peacock SJ, Witney AA et al. (2006) Microarrays reveal that each of the ten dominant lineages of Staphylococcus aureus has a unique combination of surfaceassociated and regulatory genes. J Bacteriol 188: 669-676. doi: 10.1128/JB.188.2.669-676.2006. PubMed: 16385056

10. Blomfeldt A, Aamot HV, Eskesen AN, Müller F, Monecke S (2013) Molecular Characterization of Methicillin-Sensitive Staphylococcus aureus Isolates from Bacteremic Patients in a Norwegian University Hospital. J Clin Microbiol 51: 345-347. doi:10.1128/JCM.02571-12. PubMed: 23135934.

11. Chi CY, Wang SM, Lin CC, Liu CC (2010) Microbiological characteristics of community-associated Staphylococcus aureus causing uncomplicated bacteremia and infective endocarditis. J Clin Microbiol 48: 292-294. doi:10.1128/JCM.01788-09. PubMed: 19846649.

12. Tristan A, Ying L, Bes M, Etienne J, Vandenesch F et al. (2003) Use of multiplex PCR to identify Staphylococcus aureus adhesins involved in human hematogenous infections. J Clin Microbiol 41: 4465-4467. doi: 10.1128/JCM.41.9.4465-4467.2003. PubMed: 12958296.

13. Moreillon P, Entenza JM, Francioli $P$, McDevitt D, Foster TJ et al. (1995) Role of Staphylococcus aureus coagulase and clumping factor in pathogenesis of experimental endocarditis. Infect Immun 63: 4738-4743. PubMed: 7591130

14. Que YA, François $P$, Haefliger JA, Entenza JM, Vaudaux $P$ et al. (2001) Reassessing the role of Staphylococcus aureus clumping factor and fibronectin-binding protein by expression in Lactococcus lactis. Infect Immun 69: 6296-6302. doi:10.1128/IAI.69.10.6296-6302.2001. PubMed: 11553573

15. Que YA, Haefliger JA, Piroth L, François P, Widmer E et al. (2005) Fibrinogen and fibronectin binding cooperate for valve infection and invasion in Staphylococcus aureus experimental endocarditis. J Exp Med 201: 1627-1635. doi:10.1084/jem.20050125. PubMed: 15897276.

16. Söderquist $B$, Kanclerski K, Sundqvist KG, Colque-Navarro $P$, Holmberg $\mathrm{H}$ et al. (1998) Cytokine response to staphylococcal exotoxins in Staphylococcus aureus septicemia. Clin Microbiol Infect 4: 366-372. doi:10.1111/j.1469-0691.1998.tb00080.x. PubMed: 11864351.

17. Li JS, Sexton DJ, Mick N, Nettles R, Fowler VG Jr. et al. (2000) Proposed modifications to the Duke criteria for the diagnosis of infective endocarditis. Clin Infect Dis 30: 633-638. doi:10.1086/313753. PubMed: 10770721

18. Monecke S, Jatzwauk L, Weber S, Slickers P, Ehricht R (2008) DNA microarray-based genotyping of methicillin-resistant Staphylococcus aureus strains from Eastern Saxony. Clin Microbiol Infect 14: 534-545. doi:10.1111/j.1469-0691.2008.01986.x. PubMed: 18373691.

19. Monecke S, Slickers P, Ehricht R (2008) Assignment of Staphylococcus aureus isolates to clonal complexes based on microarray analysis and pattern recognition. FEMS Immunol Med Microbiol 53: 237-251. doi:10.1111/j.1574-695X.2008.00426.x. PubMed: 18507678.

20. Monecke S, Coombs G, Shore AC, Coleman DC, Akpaka P et al. (2011) A field guide to pandemic, epidemic and sporadic clones of methicillin-resistant Staphylococcus aureus. PLOS ONE 6: e17936. doi: 10.1371/journal.pone.0017936. PubMed: 21494333.

21. Enright MC, Day NP, Davies CE, Peacock SJ, Spratt BG (2000) Multilocus sequence typing for characterization of methicillin-resistant and methicillin-susceptible clones of Staphylococcus aureus. J Clin Microbiol 38: 1008-1015. PubMed: 10698988.

22. Melles DC, Gorkink RF, Boelens HA, Snijders SV, Peeters JK et al. (2004) Natural population dynamics and expansion of pathogenic clones of Staphylococcus aureus. J Clin Invest 114: 1732-1740. doi: 10.1172/JCI200423083. PubMed: 15599398.

23. O'Riordan K, Lee JC (2004) Staphylococcus aureus capsular polysaccharides. Clin Microbiol Rev 17: 218-234. doi:10.1128/CMR. 17.1.218-234.2004. PubMed: 14726462

24. Watts A, Ke D, Wang Q, Pillay A, Nicholson-Weller A et al. (2005) Staphylococcus aureus strains that express serotype 5 or serotype 8 capsular polysaccharides differ in virulence. Infect Immun 73: 3502-3511. doi:10.1128/IAI.73.6.3502-3511.2005. PubMed: 15908379.

25. Hogevik H, Söderquist B, Tung HS, Olaison L, Westberg A et al. (1998) Virulence factors of Staphylococcus aureus strains causing infective endocarditis--a comparison with strains from skin infections. APMIS 106: 901-908. doi:10.1111/j.1699-0463.1998.tb00237.x. PubMed: 9808417.

26. Roche FM, Massey R, Peacock SJ, Day NP, Visai L et al. (2003) Characterization of novel LPXTG-containing proteins of Staphylococcus aureus identified from genome sequences. Microbiology 149: 643-654. doi:10.1099/mic.0.25996-0. PubMed: 12634333.

27. Peacock SJ, Day NP, Thomas MG, Berendt AR, Foster TJ (2000) Clinical isolates of Staphylococcus aureus exhibit diversity in fnb genes and adhesion to human fibronectin. J Infect 41: 23-31. doi:10.1053/jinf. 2000.0657. PubMed: 10942636.

28. Heilmann C, Niemann S, Sinha B, Herrmann M, Kehrel BE et al. (2004) Staphylococcus aureus fibronectin-binding protein (FnBP)-mediated adherence to platelets, and aggregation of platelets induced by FnBPA but not by FnBPB. J Infect Dis 190: 321-329. doi:10.1086/421914. PubMed: 15216468

29. Corrigan RM, Rigby D, Handley P, Foster TJ (2007) The role of Staphylococcus aureus surface protein SasG in adherence and biofilm formation. Microbiology 153: 2435-2446. doi:10.1099/mic. 0.2007/006676-0. PubMed: 17660408.

30. Werbick C, Becker K, Mellmann A, Juuti KM, von Eiff C et al. (2007) Staphylococcal chromosomal cassette mec type I, spa type, and expression of Pls are determinants of reduced cellular invasiveness of methicillin-resistant Staphylococcus aureus isolates. J Infect Dis 195: 1678-1685. doi:10.1086/517517. PubMed: 17471438.

31. Xiong YQ, Fowler VG, Yeaman MR, Perdreau-Remington F, Kreiswirth BN et al. (2009) Phenotypic and genotypic characteristics of persistent methicillin-resistant Staphylococcus aureus bacteremia in vitro and in an experimental endocarditis model. J Infect Dis 199: 201-208. doi: 10.1086/595738. PubMed: 19086913.

32. Wehrhahn MC, Robinson JO, Pascoe EM, Coombs GW, Pearson JC et al. (2012) Illness severity in community-onset invasive Staphylococcus aureus infection and the presence of virulence genes. J Infect Dis 205: 1840-1848. doi:10.1093/infdis/jis279. PubMed: 22492857.

33. Gillaspy AF, Lee CY, Sau S, Cheung AL, Smeltzer MS (1998) Factors affecting the collagen binding capacity of Staphylococcus aureus. Infect Immun 66: 3170-3178. PubMed: 9632582.

34. Sabat A, Melles DC, Martirosian G, Grundmann H, van Belkum A et al. (2006) Distribution of the serine-aspartate repeat protein-encoding sdr genes among nasal-carriage and invasive Staphylococcus aureus strains. J Clin Microbiol 44: 1135-1138. doi:10.1128/JCM. 44.3.1135-1138.2006. PubMed: 16517913.

35. Cucarella C, Solano C, Valle J, Amorena B, Lasa I et al. (2001) Bap, a Staphylococcus aureus surface protein involved in biofilm formation. J Bacteriol 183: 2888-2896. doi:10.1128/JB.183.9.2888-2896.2001. PubMed: 11292810.

36. van Belkum A, Melles DC, Snijders SV, van Leeuwen WB, Wertheim $\mathrm{HF}$ et al. (2006) Clonal distribution and differential occurrence of the 
enterotoxin gene cluster, egc, in carriage- versus bacteremiaassociated isolates of Staphylococcus aureus. J Clin Microbiol 44: 1555-1557. doi:10.1128/JCM.44.4.1555-1557.2006. PubMed: 16597892.

37. von Eiff C, Friedrich AW, Peters G, Becker K (2004) Prevalence of genes encoding for members of the staphylococcal leukotoxin family among clinical isolates of Staphylococcus aureus. Diagn Microbiol Infect Dis 49: 157-162. doi:10.1016/j.diagmicrobio.2004.03.009. PubMed: 15246504. 